\title{
Basic Study of IPMSM with High-Temperature Superconducting Wire Rod
}

\author{
Kazuya Okada*, Shigeo Morimoto*, Masayuki Sanada* and Yukinori Inoue*
}

\begin{abstract}
It is important to improve the efficiencies of motors to overcome problems such as decreasing energy reserves and environmental pollution. Superconductors are promising for developing high-efficiency motors. However, superconducting wires must be kept in critical conditions and the AC loss needs to be minimized. In this paper, a design of a superconducting interior permanent magnet synchronous motor (IPMSM) is proposed that reduces the AC loss. The characteristics of superconducting and normal-conducting IPMSMs are compared. The proposed superconducting IPMSM has a low AC loss and a very high efficiency at low speeds.
\end{abstract}

Keywords: IPMSM, Superconducting, HTS motor, AC loss

\section{Introduction}

In recent years, much effort has gone into reducing energy consumption to overcome problems with decreasing energy reserves and environmental issues. One promising way to achieve this is to reduce the energy consumption of motors by improving their efficiency. Energy losses in motors are mainly due to copper and iron losses. Copper loss occurs due to Joule heating caused by the winding resistance when a current flows, while iron loss is due to hysteresis and eddy current losses when the magnetic material in the motor is magnetized by the $\mathrm{AC}$ magnetic field. Various studies have sought to reduce these losses to realize high-efficiency motors. A promising means to achieve this are superconducting motors containing a hightemperature superconductor (HTS). Most HTS motors use superconducting magnets as rotor poles. However, this study discusses a HTS interior permanent magnet synchronous motor (IPMSM) with superconducting armature coils [1]-[5].

Since superconducting wires have no electrical resistance and can withstand high current densities, they can generate high magnetic fields with no copper loss. However, they must be kept in critical conditions. In addition, AC loss occurs instead of copper loss when an AC current flows through superconducting wires. AC loss is caused by the varying magnetic field applied to the superconducting

\footnotetext{
* Dept. of Electrical and Information Systems, Osaka Prefecture University, Japan. (s0110okada@eis.osakafu-u.ac.jp,morimoto@eis. osakafu-u.ac.jp, inoue@eis.osakafu-u.ac.jp, sanada@eis.osakafu-u. ac.jp)
}

Received 10 April 2013 ; Accepted 1 May 2013 armature winding. Thus, designs seek to minimize the AC loss by considering the flux flow.

In this paper, a design for a superconducting IPMSM that reduces AC loss is proposed and the characteristics of superconducting and normal-conducting with the same rotor and stator core are compared. Moreover, a suitable design is proposed for realizing a high torque and a high output power from a superconducting IPMSM.

\section{Design for AC Loss Reduction}

\subsection{Analysis Model}

Fig. 1 shows the analysis model used in this study and Table 1 lists the motor specifications. The analysis model has four poles and six slots. Concentrated winding is used in the stator. As shown in Fig. 1(b), the refrigerant circulates through a slot to cool the superconducting wire and the wire is surrounded by a vacuum to insulate it. This IPMSM was analyzed by 2-D finite-element method.

\subsection{Position and Speed Estimation Method}

When using superconducting wire for the stator winding of an $\mathrm{AC}$ motor, it is necessary to consider the critical current and $\mathrm{AC}$ loss characteristics. The critical current decreases with increasing magnetic field applied to the superconducting wire. Consequently, it is necessary to carefully consider the amplitude of the magnetic field applied to the superconducting wire. On the other hand, AC loss occurs when a varying magnetic field is applied to the superconducting wire. The AC loss is strongly affected by a 


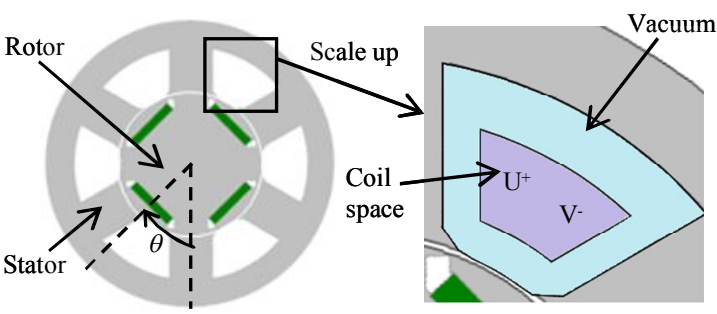

(a) Sectional view

(b) Slot part

Fig. 1. Motor structure of analysis model

Table 1. Specifications of Analysis Model

\begin{tabular}{|c|c|}
\hline Item (Unit) & Value \\
\hline Stator outer diameter $(\mathrm{mm})$ & 470 \\
\hline Rotor outer diameter $(\mathrm{mm})$ & 238 \\
\hline Air gap length $(\mathrm{mm})$ & 1.0 \\
\hline Stack length $(\mathrm{mm})$ & 130 \\
\hline Number of turns (turns/phase) & 90 \\
\hline Coercive force of PM (kA/m) & 1,034 \\
\hline Rated current $I_{e m}(\mathrm{~A})$ & 500 \\
\hline Voltage limitation $V_{a m}(\mathrm{~V})$ & 283 \\
\hline Maximum speed $\left(\mathrm{min}^{-1}\right)$ & 2,500 \\
\hline
\end{tabular}

changing magnetic field component in the thickness direction (i.e., the perpendicular component) due to anisotropic electromagnetic properties of superconductors. The influence of the perpendicular magnetic field on the AC loss increases with increasing applied magnetic field; at a high applied magnetic field, the AC loss generated by a perpendicular magnetic field is more than 10 times greater than that caused by the horizontal magnetic field.

This study proposes a design of a superconducting IPMSM that reduces the AC loss by reducing the perpendicular magnetic field at the superconducting wire. To reduce the perpendicular magnetic field, the coil is configured such that the superconducting wire is oriented parallel to the magnetic field applied to it and it uses a stator with steel in the slot. Fig. 2 shows how the perpendicular magnetic field is reduced by inclining the superconducting wire. As shown in Fig. 2, the perpendicular magnetic field can be reduced by orienting the superconducting wire parallel to the magnetic field.

\subsection{Calculation and Result of AC Loss Reduction}

The AC loss was calculated by magnetic field analysis. In this analysis, the magnetic field at each element in the cross section of the superconducting wire is decomposed into perpendicular and horizontal components and the AC loss is then calculated by summing the losses caused by

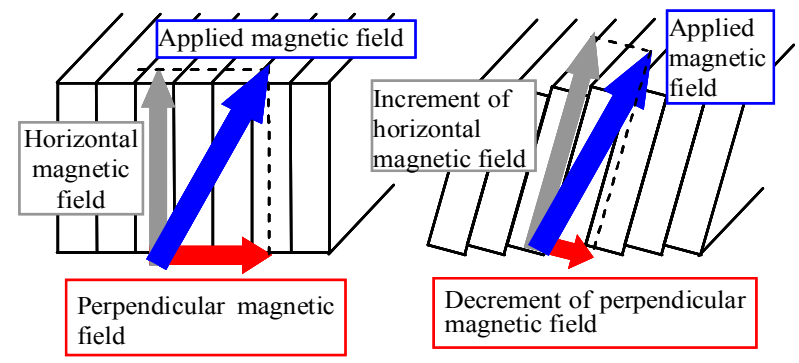

Fig. 2. Reduction of perpendicular magnetic field by inclining coil

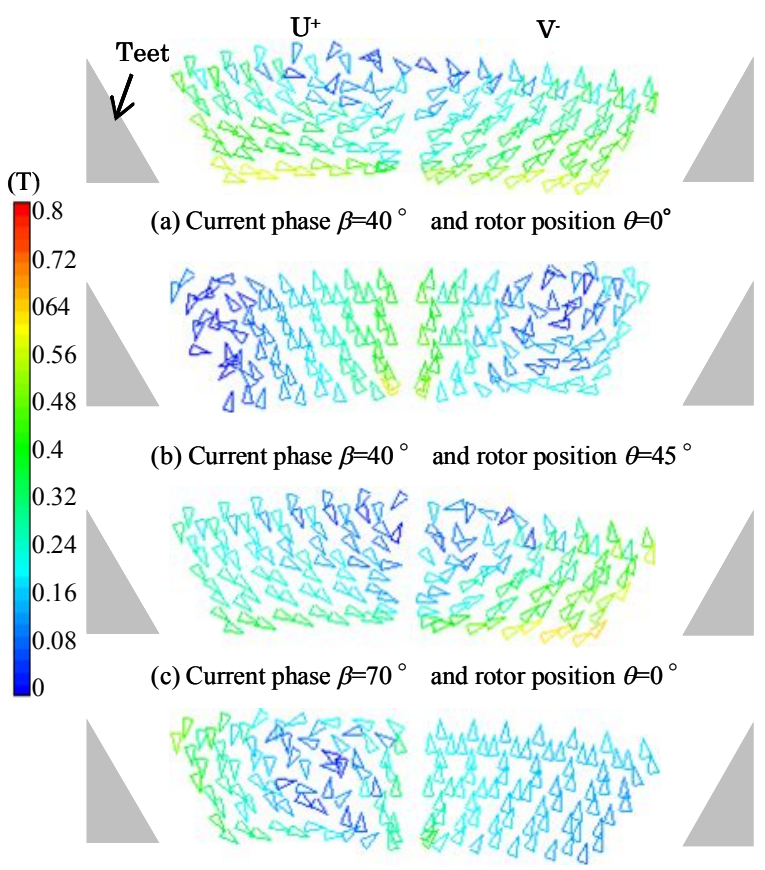

(d) Current phase $\beta=70^{\circ}$ and rotor position $\theta=45^{\circ}$

Fig. 3. Flux density vector of coil (500A)

each magnetic field component at all elements.

Fig. 3 shows the flux density vector of the coil for a stator current of $500 \mathrm{~A}$ under various conditions. It shows that the sizes and directions of the flux density vectors vary with the current phase $\beta$ (defined as the leading electrical angle relative to the $q$ axis) and the rotor position $\theta$ (defined as the mechanical angle relative to the direction in which the magnetic field of the $\mathrm{N}$ pole and $\mathrm{U}$ phase are aligned). Moreover, since the flux density vectors of the coils have different directions, it is necessary to determine the coil angle that minimizes the total AC loss. In this paper, this angle is determined to minimize the $\mathrm{AC}$ loss at the maximum current (500 A).

Fig. 4 shows the maximum perpendicular magnetic field of the coil as a function of the inclination angle of the coil under the conditions that maximize the torque. Here, the inclination angle of the coil is positive when the coil is 
inclined in the clockwise direction. As shown in Fig. 4, the perpendicular magnetic field decreases as the inclination angle of coil becomes increasingly positive, irrespective of whether steel is placed in the slot. Moreover, when the inclination angle exceeds $15^{\circ}$, the perpendicular magnetic field of the superconducting IPMSM with steel in the slot is smaller than that without steel in the slot. Thus, the perpendicular magnetic field can be reduced and the superconducting armature winding will be less affected by external magnetic fields by inclining the coil and placing steel in the slot.

Fig. 5 shows the inclination angle of the coil as a function of the $\mathrm{AC}$ loss characteristics for four current phases. The AC loss is normalized by that at an inclination angle of $30^{\circ}$. Fig. 5 shows that, at all four current phases, the AC loss increases (decreases) as the inclination angle of the coil becomes more negative (positive). Moreover, the ratio of the $\mathrm{AC}$ loss reduction increases with increasing current phase. The coil inclination angle that minimizes the $\mathrm{AC}$ loss varies for each current phase; it thus should be determined base on the application of the motor. In the present analysis model, an inclination angle of $30^{\circ}$ minimized the AC loss at the maximum current.

Fig. 6 shows the AC loss as a function of the speed for when steel is and is not placed in the slot. Here, the AC loss was calculated for coil inclination angle of $30^{\circ}$. Fig. 6 shows that the AC loss increases with increasing speed. Inserting steel in the slot reduces the $\mathrm{AC}$ loss at each current; in particular, the difference in the AC loss at high speeds is larger than that at low speeds. This result suggests that inserting steel in the slot reduces the AC loss more at higher current phases.

\section{Performance of Superconducting IPMSM}

Fig. 7 shows the output power as a function of speed in maximum power control that combines maximum torque/ampere control below the base speed and fluxweakening control or maximum torque/flux control above base speed [6]. Table 2 lists the analysis results. Here, $V_{a m}$ is assumed to be $283 \mathrm{~V}$. The output power is calculated by considering losses such as the AC loss, the iron loss, and the copper loss. The normal-conducting IPMSM has a copper armature winding. The normal-conducting and superconducting IPMSMs have the same rotor and stator core specifications and the space factor is 0.5 . In this case, the current density is $6.3 \mathrm{~A} / \mathrm{mm}^{2}$. As shown in Fig. 7, the output powers of the two models are almost the same below the base speed, whereas they differ slightly above the base speed. However, as shown in Table 2, since the maximum $d$-axis armature reaction $L_{d} I_{a m}$ is much larger than the

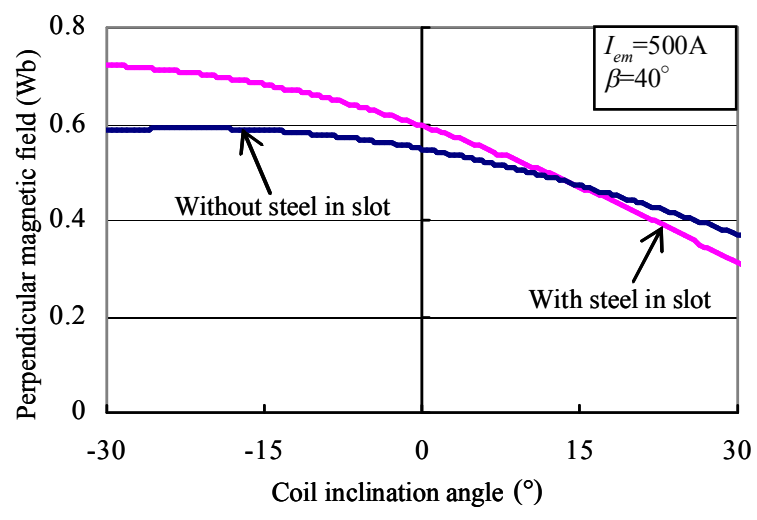

Fig. 4. Perpendicular magnetic field vs. inclination angle

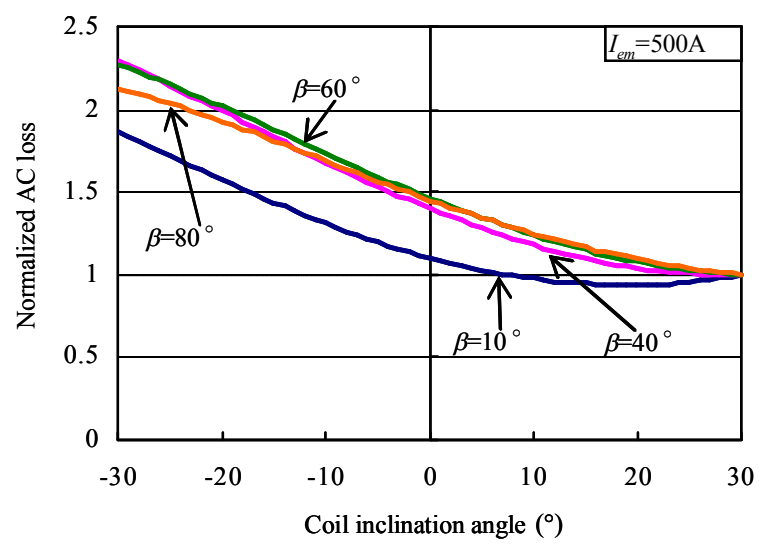

Fig. 5. AC loss vs. inclination angle

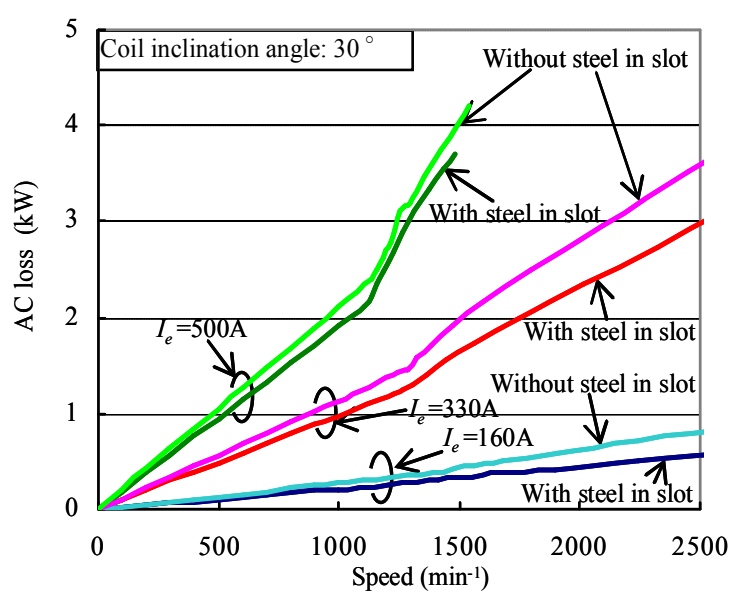

Fig. 6. AC loss vs. speed characteristics

armature flux linkage of the PM $\Psi_{a}$, the minimum $d$-axis flux linkage $\Psi_{d \min }$ is negative. Thus, the two models do not have constant output power characteristics above the base speed and have low power factors. Therefore, it is important to realize a constant output power and a high power factor when designing a superconducting IPMSM.

Fig. 8 shows the loss characteristics for maximum power 


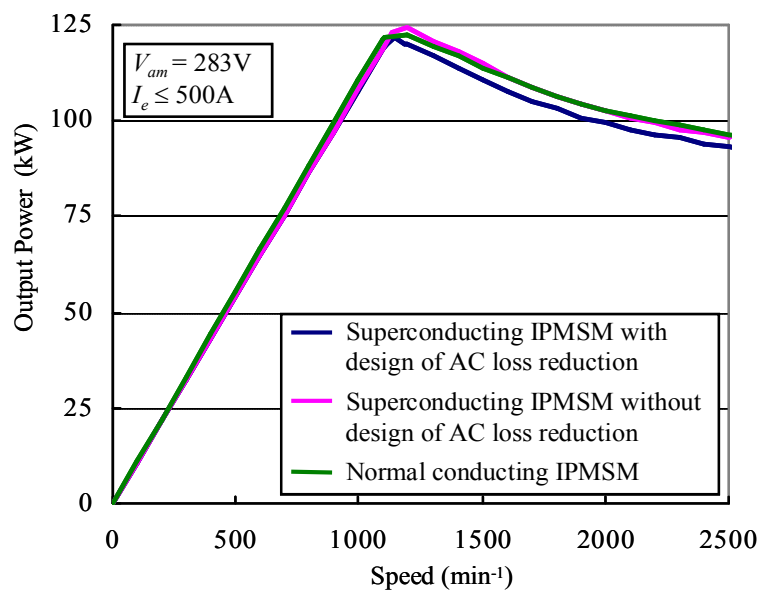

Fig. 7. Output power vs. speed characteristics

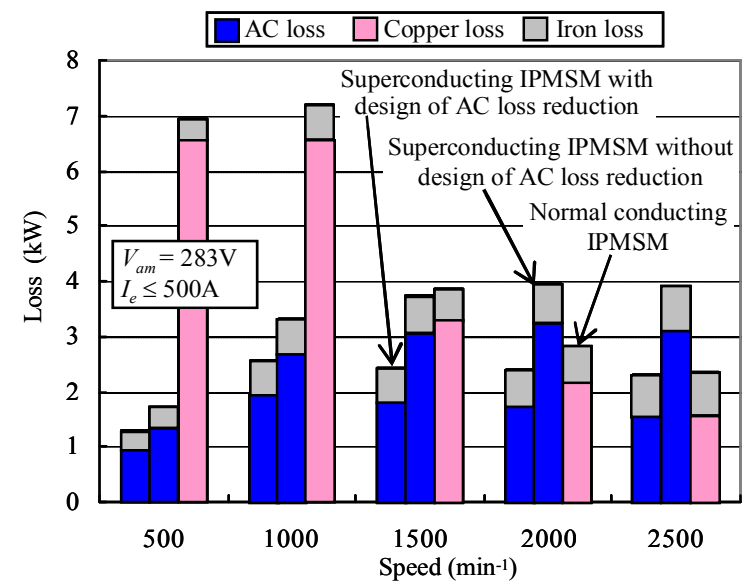

Fig. 8. Loss vs. speed characteristics

control. The iron loss was calculated using the following equations.

$$
\begin{array}{r}
P_{r-i}=\sum_{k=1}^{n}\left\{\varepsilon \cdot k \cdot f \cdot B_{r-i, k}^{2}+\sigma\left(k \cdot f \cdot B_{r-i}, k\right)^{2}\right\} \\
P_{\theta-i}=\sum_{k=1}^{n}\left\{\varepsilon \cdot k \cdot f \cdot B_{\theta-i, k}^{2}+\sigma\left(k \cdot f \cdot B_{\theta-i}, k\right)^{2}\right\} \\
W_{\text {iron }}=\sum_{i=1}^{N} m_{i}\left(P_{r-i}+P_{\theta-i}\right)
\end{array}
$$

where $P_{r-i}$ is the iron loss due to the radial flux of the $i$ th element in the finite-element model, $i$ is the element number, $k$ is the harmonic order, $f$ is the fundamental frequency, $\varepsilon$ is the hysteresis loss coefficient, $\sigma$ is the eddy current loss coefficient, $B_{r-i, k}$ is the $k$ th harmonic component of the magnetic flux density in the radial

\begin{tabular}{|c|c|c|c|}
\hline \multirow[b]{2}{*}{ Item (Unit) } & \multicolumn{2}{|c|}{ Superconducting IPMSM } & \multirow{2}{*}{$\begin{array}{l}\text { Normal- } \\
\text { Conducting } \\
\text { IPMSM }\end{array}$} \\
\hline & $\begin{array}{l}\text { With design } \\
\text { of } \mathrm{AC} \text { loss } \\
\text { reduction }\end{array}$ & $\begin{array}{l}\text { Without design } \\
\text { of } \mathrm{AC} \text { loss } \\
\text { reduction }\end{array}$ & \\
\hline \begin{tabular}{|c|} 
Average torque \\
$(\mathrm{Nm})$
\end{tabular} & 1,055 & 1,062 & 1,060 \\
\hline $\begin{array}{l}\text { Base speed } \\
\left(\min ^{-1}\right)\end{array}$ & 1,114 & 1,139 & 1,104 \\
\hline$\Psi_{a}(\mathrm{~Wb})$ & 0.412 & 0.423 & 0.413 \\
\hline$L_{d} I_{a m}(\mathrm{~Wb})$ & 1.319 & 1.299 & 1.304 \\
\hline $\begin{array}{c}\Psi_{d \min }(\mathrm{Wb}) \\
\left(=\Psi_{a}-L_{d} I_{a m}\right)\end{array}$ & -0.907 & -0.876 & -0.891 \\
\hline $\begin{array}{l}\text { Power factor } \\
\text { at maximum } \\
\text { output power }\end{array}$ & 0.513 & 0.527 & 0.517 \\
\hline
\end{tabular}
direction of the $i$ th element, $P_{\theta-i}$ is the iron loss due to the
Table 2. Analysis Results

tangential flux at the $i$ th element, $B_{r-i, k}$ is the $k$ th harmonic component of the magnetic flux density in the tangential direction of the $i$ th element, $N$ is the total number of elements, $m_{i}$ is the mass of the $i$ th element, and $W_{\text {iron }}$ is the total iron loss.

As shown in Fig. 8, the AC loss below the base speed increases with increasing speed, whereas the AC and copper losses above the base speed decrease with increasing speed due to a reduction in the current for maximum torque/flux control. The AC loss decreases by about $30 \%$ below the base speed and by about $50 \%$ above the base speed in the design to reduce the AC loss. In addition, the total loss of the superconducting IPMSM that is designed to minimize the AC loss is smaller than that of the normal-conducting IPMSM at all speeds (but especially below the base speed). However, there is little difference between the AC loss and the copper loss at high speeds, so other approaches to reduce the AC loss have to be considered.

Fig. 9 shows the efficiency vs. speed for maximum output control. It shows that the superconducting model has a much higher efficiency below the base speed than the normal-conducting model since it does not have any copper loss. Moreover, in the design that reduces the AC loss, the superconducting IPMSM is more efficient than the normalconducting IPMSM. However, since the copper loss decreases more than the AC loss above the base speed due to maximum torque/flux control, the efficiencies of the superconducting and normal-conducting models do not differ by much.

\section{Improvement of Output Power}

\subsection{Analysis Model}

Existing superconducting IPMSMs suffer from several 


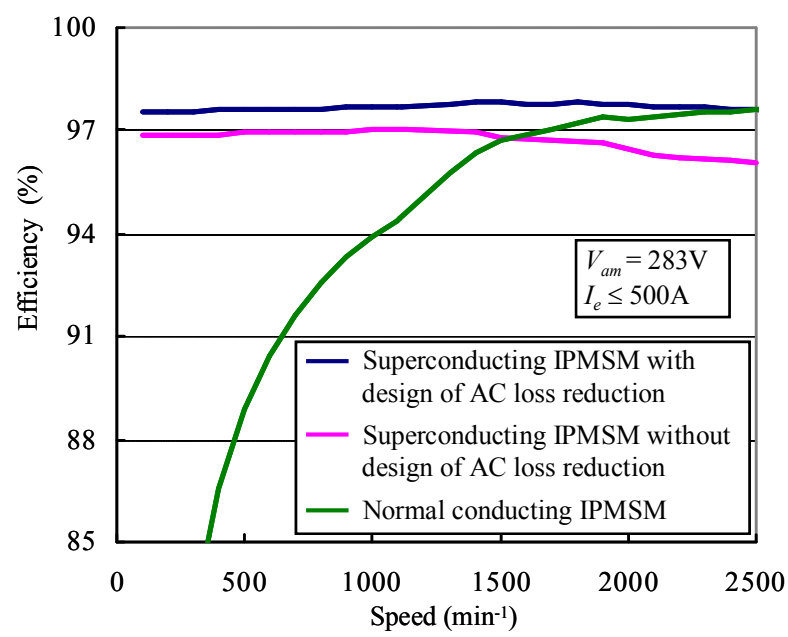

Fig. 9. Efficiency vs. speed characteristics
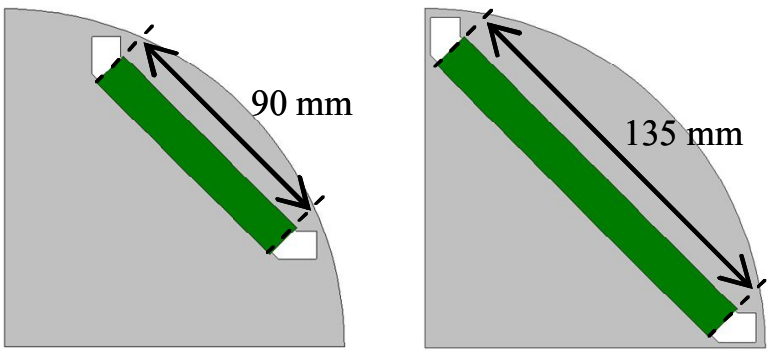

(a) PM width of $90 \mathrm{~mm}$

(b) PM width of $135 \mathrm{~mm}$

Fig. 10. Rotor structure of analysis model

problems, including reduced output power above the base speed and a low power factor. It is thus important to develop a suitable design for a superconducting IPMSM that has a high output power and a high power factor with the aim of realizing $\Psi_{d m i n}=0$. In this paper, $L_{d} I_{a m}$ is reduced by reducing the number of turns and $\Psi_{a}$ is increased by increasing the amount of the PM. Fig. 10 shows the rotor structure of this analysis model and Table 3 lists the changed specifications of this analysis model. Fig. 10(a) is the same as Fig. 1. As shown in Fig. 10(b), PM increases only in the width direction. In addition, the stator inner diameter and the rotor outer diameter are increased due to the reduced number of turns.

\subsection{Analysis Results}

Table 4 shows the analysis results for the flux linkage and Fig. 11 shows the output power vs. speed. Table 4 shows that $L_{d} I_{a m}$ decreases with decreasing number of turns and that $\Psi_{d \min }$ approaches zero. In addition, Fig. 11 shows that the maximum output power and the output power above the base speed both increase with decreasing number of turns. In addition, $\Psi_{d m i n}$ is closer to zero due to the
Table 3. Motor Diameter and Number of Tutns

\begin{tabular}{|c|r|r|r|}
\hline Item (Unit) & \multicolumn{3}{|c|}{ Value } \\
\hline $\begin{array}{c}\text { Number of turns } \\
\text { (turns/phase) }\end{array}$ & 90 & 64 & 34 \\
\hline $\begin{array}{c}\text { Stator inner diameter } \\
\text { (mm) }\end{array}$ & 240 & 250 & 260 \\
\hline $\begin{array}{c}\text { Rotor outer diameter } \\
\text { (mm) }\end{array}$ & 238 & 248 & 258 \\
\hline Width of PM (mm) & \multicolumn{3}{|c|}{90,135} \\
\hline
\end{tabular}

Table 4. Analysis Result of Flux Linkage

\begin{tabular}{|c|c|c|c|c|c|c|}
\hline $\begin{array}{c}\text { Width of PM } \\
(\mathrm{mm})\end{array}$ & \multicolumn{3}{|c|}{90} & \multicolumn{3}{c|}{135} \\
\hline $\begin{array}{c}\text { Number of } \\
\text { turns } \\
\text { (turns/phase) }\end{array}$ & 90 & 64 & 34 & 90 & 64 & 34 \\
\hline$\Psi_{a}(\mathrm{~Wb})$ & 0.421 & 0.346 & 0.213 & 0.592 & 0.481 & 0.298 \\
\hline$L_{d} I_{a m}(\mathrm{~Wb})$ & 1.319 & 0.746 & 0.245 & 1.372 & 0.736 & 0.221 \\
\hline$\Psi_{d \min }(\mathrm{Wb})$ & -0.897 & -0.400 & -0.032 & -0.780 & -0.256 & 0.078 \\
\hline
\end{tabular}

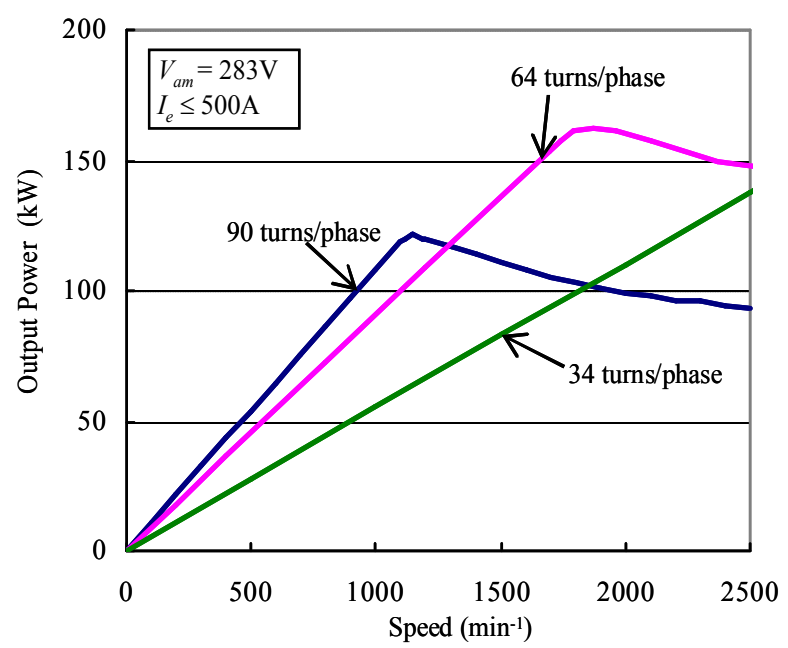

(a) PM Width of $90 \mathrm{~mm}$

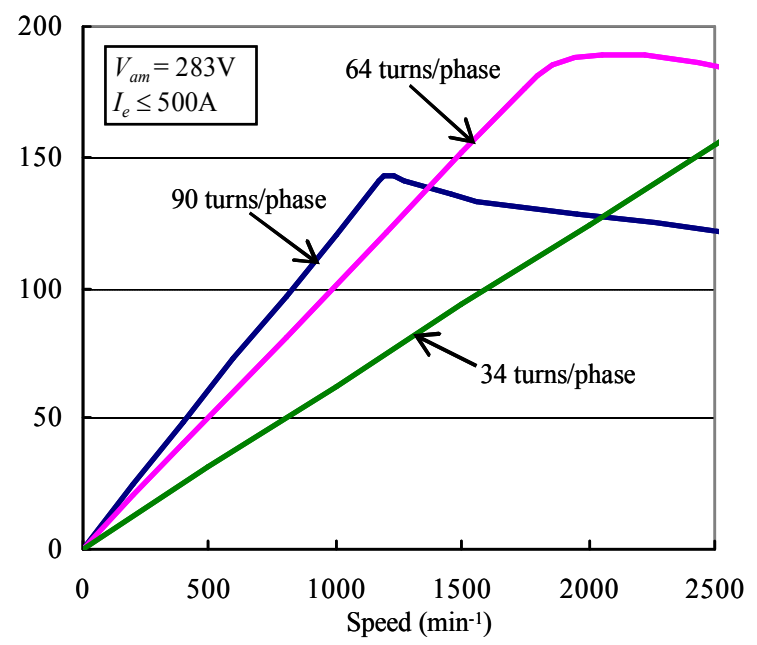

(b) PM Width of $135 \mathrm{~mm}$

Fig. 11. Output power vs. speed characteristics 
increase in $\Psi_{a}$ on increasing the amount of PM. Consequently, the torque and the output power increase more and the decline in the output power becomes slower. In this study, the model with 34 turns/phase exhibits the largest output power and power factor, but it has a much smaller torque than the other models and it does not approach the maximum output power below the maximum speed. Therefore, the model with 64 turns/phase and a PM width of $135 \mathrm{~mm}$ is considered to be the best model examined in this study.

\section{Conclusion}

In an IPMSM with superconducting wires that is designed to reduce the AC loss by using an inclined coil and steel in a slot was proposed. The characteristics of superconducting and normal-conducting IPMSMs were compared. The results indicate that the AC loss is reduced by more than $30 \%$ at low speeds and by $50 \%$ at high speeds due to the design to reduce the AC loss. Superconducting and normal-conducting IPMSMs are expected to have similar characteristics with the exception of their efficiency vs. speed characteristics. However, the superconducting IPMSM is more efficient than the normal-conducting IPMSM.

The superconducting IPMSM suffers from some problems including a reduced output power at high speeds. To improve the output power characteristics of the superconducting IPMSM, the number of turns was reduced and the amount of PM was increased. The results indicate that the maximum output power is increased and the decline in the output power is slower.

\section{References}

[1] T. Baba, H. Oyama, T. Ariyoshi and T. Sawai, "Design of Superconducting Motor" SEI technical review Vol.176 pp.45-50, 2010.

[2] H. Wen, W. Bailey, K. Goddard, M. Al-Mosawi, C. Beduz, Y. Yang, "Performance Test of a 100kW HTS Generator Operating at 67 K-77 K" IEEE Transactions on Applied Superconductivity, Vol.19, no.3, pp.1652-1655, 2009.

[3] Y. K. Kwon, M. H. Sohn, S. K. Baik, E. Y. Lee, J. M. Kim, T. S. Moon, H. J. Park, Y. C. Kim, K. S. Ryu, "Development of a $100 \mathrm{hp} \mathrm{Synchronous} \mathrm{Motor} \mathrm{With} \mathrm{HTS} \mathrm{Field} \mathrm{Coils"} \mathrm{IEEE}$ Transactions on Applied Superconductivity, Vol. 15, no.2, pp.2194-2197, 2005.

[4] F. Xu, A. Chen, S. Yang, J. Cao, X. Liu, L. Li, "AC Loss Prediction in BSCCO-Tape Armature Winding Design of a Synchronous Motor" IEEE Transactions on Applied Superconductivity, Vol.20, no.3, pp.1005-1008, 2010.

[5] L. Li, J. Cao, Z. Sun, Q. Chen, "Magnetic Field Distribution Around Superconducting Coil in Ferromagnetic Environment" IEEE Transactions on Applied Superconductivity, Vol.21, no.3, pp.1131-1135, 2011.
[6] S. Morimoto, Y. Takeda, T. Hirasa, and K. Taniguchi, "Expansion of Operating Limits for Permanent Magnet Motor by Current Vector Control Considering Inverter Capacity," IEEE Trans. Ind. Applicat., vol. 26, No.5, pp. 866-871, 1990.

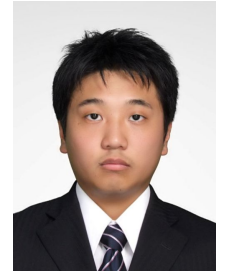

Kazuya Okada received the B.E. and M.E. degrees from Osaka Prefecture University, in 2011 and 2013, respectively. He was involved in the study on the hightemperature superconducting IPMSM at Osaka Prefecture University. $\mathrm{He}$ is currently with Hitachi Automotive Systems, Ltd., Tokyo, Japan.

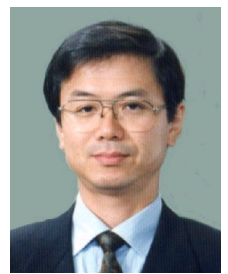

Shigeo Morimoto received the B.E., M.E., and $\mathrm{Ph} . \mathrm{D}$. degrees from Osaka Prefecture University, in 1982, 1984, and 1990, respectively. Since 1988, he has been with the Graduate School of Engineering, Osaka Prefecture University, where he is currently a Professor. His research interests include permanent magnet synchronous machines, reluctance machines and their control systems.

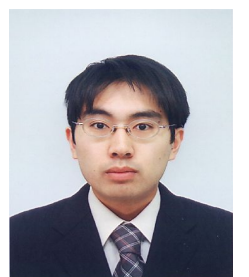

Yukinori Inoue received the B.E., M.E., and Ph.D. degrees from Osaka Prefecture University, in 2005, 2007, and 2010, respectively. Since 2010, he has been with the Graduate School of Engineering, Osaka Prefecture University, where he is currently an Assistant Professor. His research interests include control of electrical drives, particularly DTC and position-sensorless control of PMSMs.

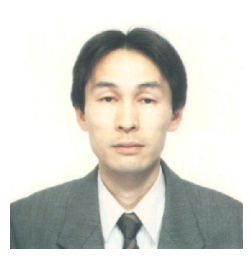

Masayuki Sanada received the B.E., M.E., and Ph.D. degrees from Osaka Prefecture University, in 1989, 1991, and 1994, respectively. Since 1994, he has been with the Graduate School of Engineering, Osaka Prefecture University, where he is currently an Associate Professor. His research interests include permanent-magnet motors for direct-drive applications, their control systems, and magnetic field analysis. 